\title{
An Efficient Real-Time Frequent Pattern Mining Technique Using Diff-Sets
}

\author{
Rajanish Dass and Ambuj Mahanti \\ Indian Institute of Management Calcutta \\ \{rajanish, am\}@iimcal.ac.in
}

\begin{abstract}
Frequent pattern mining in real-time is of increasing thrust in many business applications such as e-commerce, recommender systems, and supplychain management and group decision support systems, to name a few. A plethora of efficient algorithms have been proposed till date. However, with dense datasets, the performances of these algorithms significantly degrade. Moreover, these algorithms are not suited to respond to the real-time need. In this paper, we describe BDFS(b)-diff-sets, an algorithm to perform real-time frequent pattern mining using diff-sets. Empirical evaluations show that our algorithm can make a fair estimation of the probable frequent patterns and reaches some of the longest frequent patterns much faster than the existing algorithms.
\end{abstract}

\section{Introduction}

In recent years, business intelligence systems are playing pivotal roles in fine-tuning business goals such as improving customer retention, market penetration, profitability and efficiency. In most cases, these insights are driven by analyses of historic data. Now the issue is, if the historic data can help us make better decisions, how real-time data can improve the decision making process [1].

Frequent pattern mining for large databases of business data, such as transaction records, is of great interest in data mining and knowledge discovery [2], since its inception in 1993, by Agrawal et al. Researchers have generally focused on the frequent pattern mining, as it is complex and the search space needed for finding all frequent itemsets is huge [2]. A number of efficient algorithms have been proposed in the last few years to make this search fast and accurate. Among these, a number of effective vertical mining algorithms have been recently proposed, that usually outperforms horizontal approaches. Despite many advantages of the vertical format, the methods tend to suffer, when the tid-list cardinality gets very large as in the case of dense datasets [3]. Again, these algorithms have limited themselves to either breadth first or depth first search techniques. Hence, most of the algorithms stop only after finding the exhaustive (optimal) set of frequent itemsets and do not promise to run under user defined real-time constraints and produce some satisficing (interesting sub-optimal) solutions due to their limiting characteristics[4, 5]. 


\section{Business Issues of Real-Time Frequent Pattern Mining}

Using up-to-date information, getting rid of delays, and using speed for competitive advantage is what the real-time enterprise is about. There are numerous areas where real-time decision making plays a crucial role. These include areas like real-time customer relationship management, ,real-time supply chain management systems realtime enterprise risk and vulnerability management, real-time stock management and vendor inventory, real-time recommender systems, real-time operational management with special applications in mission critical real-time information as is used in the airlines industry, real-time intrusion and real-time fraud detection, real-time negotiations and other areas like real-time dynamic pricing and discount offering to customers in real-time. More than that, real-time data mining will have tremendous importance in areas where a real-time decision can make the difference between life and death mining patterns in medical systems.

\section{BDFS(b)-Diff-Sets: An Efficient Technique of Frequent Pattern Mining in Real-Time Using Diff-Sets}

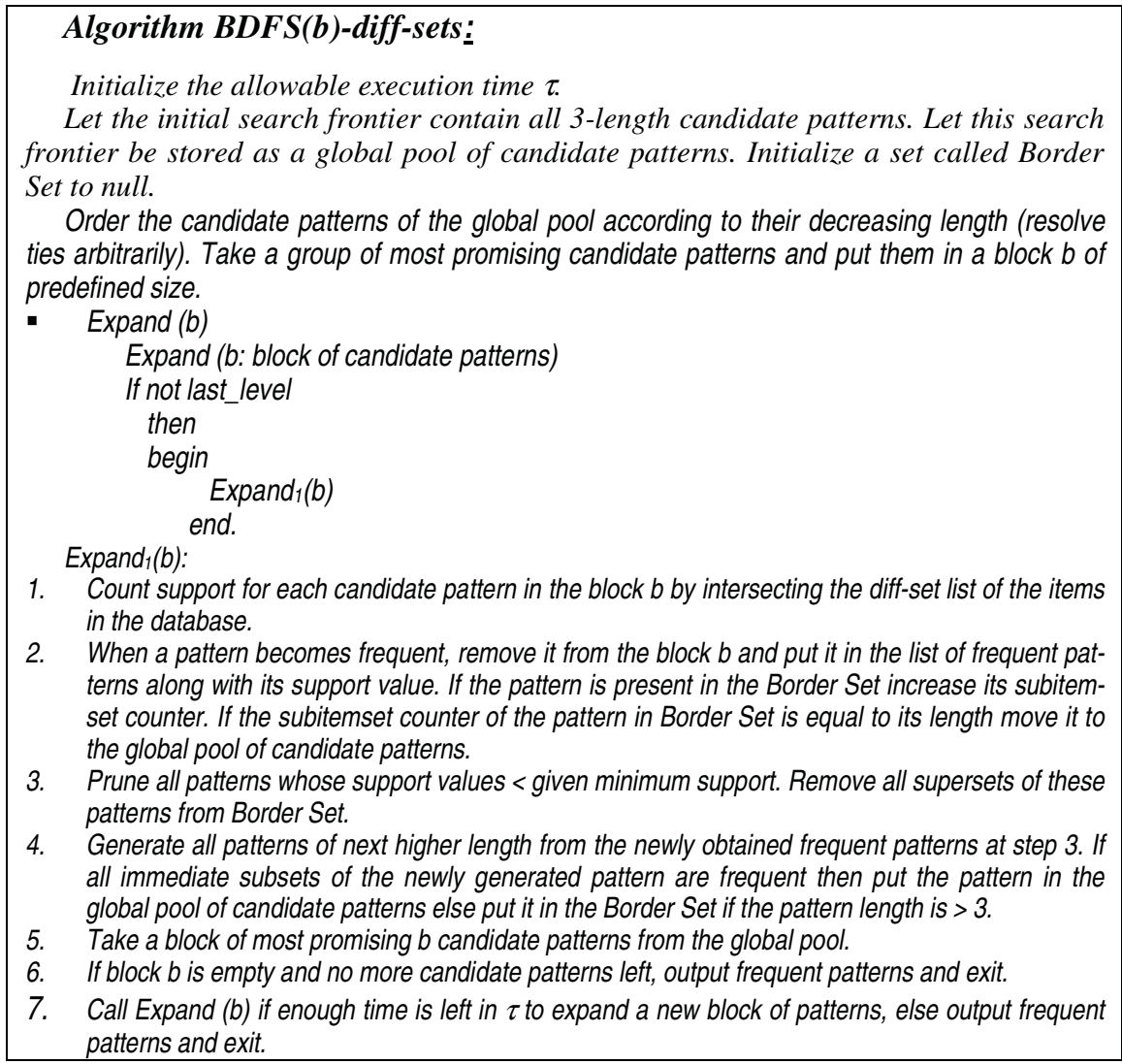

Fig. 1. Algorithm BDFS(b)-diff-sets 


\section{Empirical Evaluation}

The following figures shows the empirical evaluation of BDFS(b)-diff-sets. We have found that BDFS(b)-diff-sets compares well with the existing best performing algorithms in time of completion and scalability. Real-time performance of BDFS(b)-diffsets (in Fig. 9 and 10) show that it is always ahead of time while providing outputs. We have made detailed performance evaluation based on empirical analysis using commonly used synthetic and real-life dense datasets. Thus, we have demonstrated that real-time frequent pattern mining can be done successfully using BDFS(b)-diffsets. We believe this study will encourage use of AI heuristic search techniques in real-time frequent pattern mining.

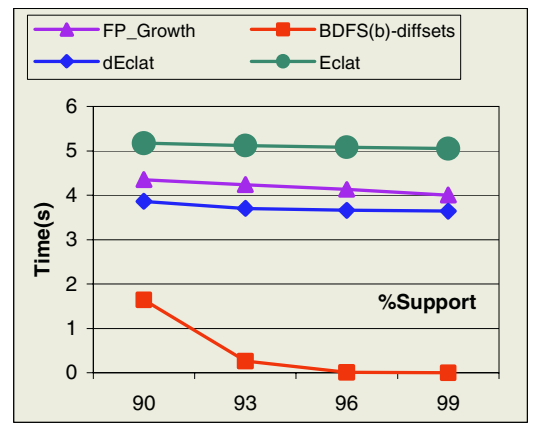

Fig. 2. Time comparison of FP-Growth, Eclat and dEclat with BDFS(b)-diffsets $(b=20880)$ on PUMSB, N=2113, T=74, $\mathrm{D}=49046$

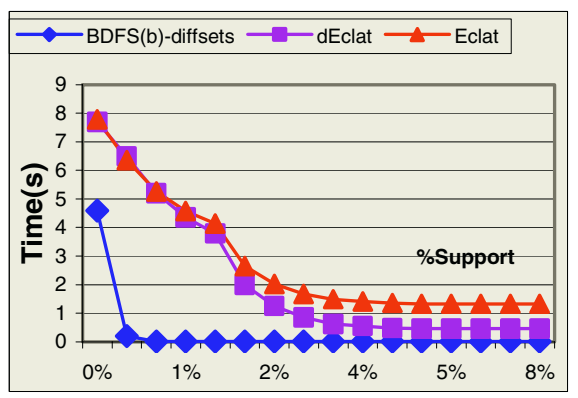

Fig. 4. Time comparison of Eclat and dEclat with BDFS(b)-diffsets for T10I8D100K, b=100K

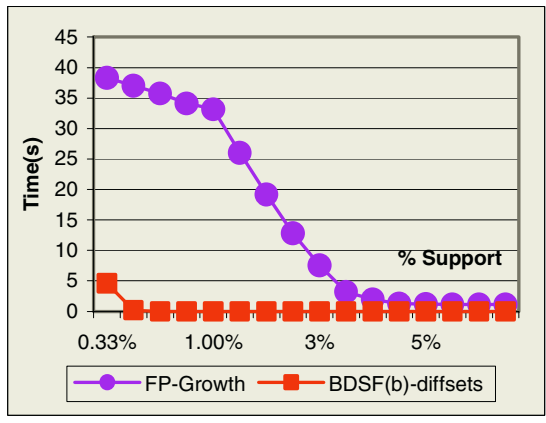

Fig. 3. Time comparison of FP-Growth with BDFS(b)-diffsets for T10I8D100K, $\mathrm{b}=100 \mathrm{~K}$

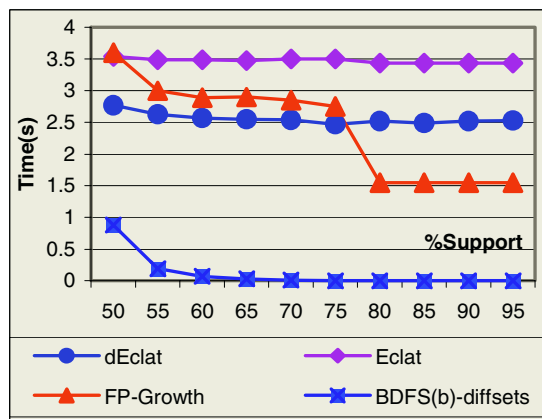

Fig. 5. Time comparison of FP-Growth, Eclat and dEclat with BDFS(b)-diffsets $(b=2088 K)$ for PUMSB*, $N=2088 \mathrm{~T}=$ 50.5, D = 49046 


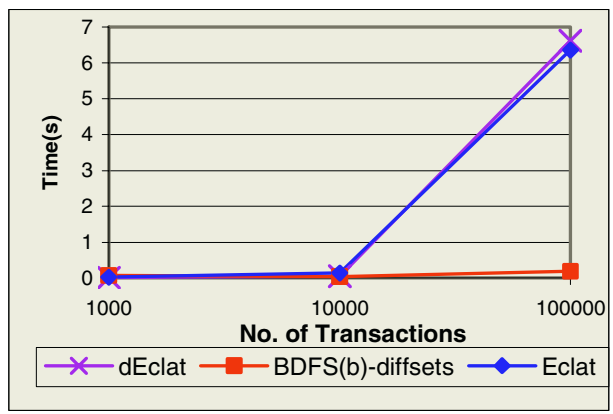

Fig. 6. Scalability evaluation of BDFS(b)-diffsets with Eclat and dEclat supp $=0.5 \%, b=100 \mathrm{~K}$ for T10I8D1K,10K and 100K

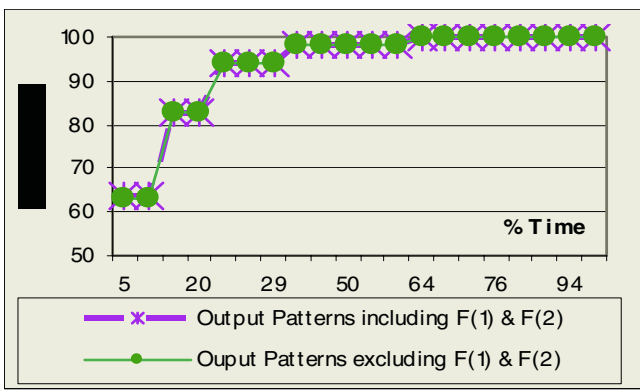

Fig. 8. Time-Patterns $\%$ of $B D F S(b)$ for $b=75 \mathrm{~K}$ and $65 \%$ supp for Chess $(\mathrm{N}=75, \mathrm{~T}=37, \mathrm{D}=3196)$

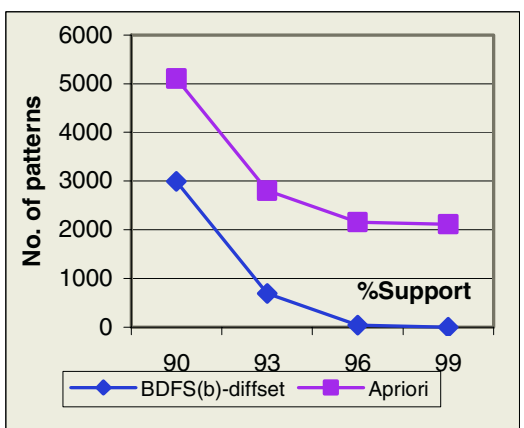

Fig. 7. Number of patterns checked by Apriori and BDFS(b)-diffsets $(b=208800)$ for Pumsb, $\mathrm{N}=2113, \mathrm{~T}=74, \mathrm{D}=49046$, with varying support

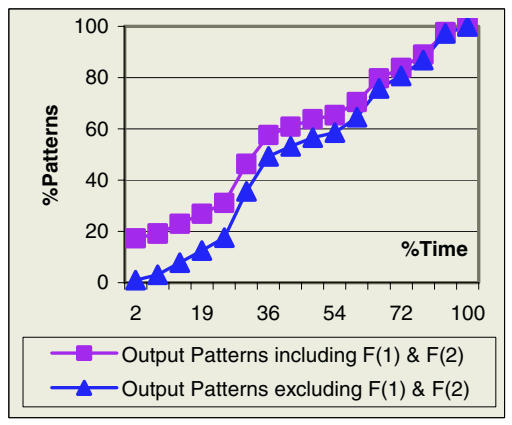

Fig. 9. Time-Patterns $\%$ for $b=75 \mathrm{~K}$ and $65 \%$ supp for T10I8D100K

\section{References}

1. Gonzales, M.L., Unearth BI in Real-time. 2004, Teradata.

2. Goethals, B., Memory Issues in Frequent Pattern Mining, in Proceedings of SAC'04. 2004, ACM: Nicosia, Cyprus.

3. Zaki, M.J. and K. Gouda. Fast Vertical Mining Using Diffsets. in 9th International Conference on Knowledge Discovery and Data Mining. 2003. Washington, DC.

4. Dass, R. and A. Mahanti. Frequent Pattern Mining in Real-Time - First Results. in TDM2004/ACM SIGKDD 2004. 2004. Seattle, Washington USA.

5. Dass, R. and A. Mahanti. An Efficient Technique for Frequent Pattern Mininig in Real-Time Business Applications. in 38th IEEE Hawaii International Conference on System Sciences (HICSS 38). 2005. Big Island: IEEE.

6. Lee, W., et al. Real time data mining-based intrusion detection. in DARPA Information Survivability Conference \& Exposition II. 2001. Anaheim, CA , USA: IEEE Xplore. 